\title{
Protocol for a systematic review of the diagnostic test accuracy of tests for IgE-mediated food allergy
}

\author{
Jon Genuneit ${ }^{1}$, Sashini Jayasinghe ${ }^{2}$, CARMEN RIGGIONI ${ }^{3}$, Rachel Peters ${ }^{4}$, Derek Chu ${ }^{5}$, \\ Daniel Munblit ${ }^{6}$, Robert Boyle ${ }^{7}$, George Du Toit ${ }^{2}$, Isabel Skypala ${ }^{8}$, and Alexandra Santos ${ }^{2}$ \\ ${ }^{1}$ Leipzig University \\ ${ }^{2}$ King's College London \\ ${ }^{3}$ Fundacion Sant Joan de Deu \\ ${ }^{4}$ Murdoch Childrens Research Institute \\ ${ }^{5}$ McMaster University \\ ${ }^{6}$ Imperial College London \\ ${ }^{7}$ Imperial College \\ ${ }^{8}$ Royal Brompton \& Harefield NHS Trust
}

July 20, 2021

\begin{abstract}
Background: The European Academy of Allergy and Clinical Immunology (EAACI) is in the process of updating the guidelines on the diagnosis and management of food allergy. The existing guidelines are based on a systematic review of the literature until 30th September 2012. Therefore, a new systematic review must be undertaken to inform the new guidelines. This systematic review aims to assess the accuracy of index tests to support the diagnosis of IgE-mediated food allergy. Methods: The databases Cochrane CENTRAL (Trials), MEDLINE (OVID) and Embase (OVID) will be searched for diagnostic test accuracy studies from 1st October 2012 to 30th June 2021. Inclusion and exclusion criteria will be used to select appropriate studies. Data from these studies will be extracted and tabulated, and then reviewed for risk of bias and applicability using the QUADAS-2 tool. All evaluation will be done in duplicate. Studies with a high risk of bias and low applicability will be excluded. Meta-analysis will be performed if there are three or more studies of the same index test and food. Results: A protocol for the systematic review and meta-analyses is presented and was registered using Prospero prior to commencing the literature search. Discussion: Oral food challenges are the reference standard for diagnosis but involve considerable risks and resources. This protocol for systematic review aims to assess the accuracy of various tests to diagnose food allergy, which can be useful in both clinical and research settings.
\end{abstract}

Protocol for a systematic review of the diagnostic test accuracy of tests for IgE-mediated food allergy

Jon Genuneit ${ }^{1}$, Sashini Jayasinghe ${ }^{2}$, Carmen Riggioni ${ }^{3}$, Rachel L. Peters ${ }^{4,5}$, Derek K. Chu ${ }^{6}$, Daniel Munblit $^{7,8}$, Robert J. Boyle ${ }^{8}$, George Du Toit ${ }^{2,9,10}$, Isabel Skypala ${ }^{11}$, Alexandra F. Santos ${ }^{2,9,10,12}$ on behalf of the EAACI Food Allergy Guidelines Expert Group and the EAACI Research and Outreach Committee Food Allergy Group*

${ }^{1}$ Pediatric Epidemiology, Department of Pediatrics, Medical Faculty, Leipzig University, Leipzig, Germany

${ }^{2}$ Department of Women and Children's Health, School of Life Course Sciences, Faculty of Life Sciences and Medicine, King's College London, London, UK 
${ }^{3}$ Allergy and Clinical Immunology, Department of Paediatrics, Yong Loo Lin School of Medicine, National University of Singapore

${ }^{4}$ Murdoch Children's Research Institute, Parkville, Victoria, Australia

${ }^{5}$ Department of Paediatrics, The University of Melbourne, Parkville, Victoria, Australia

${ }^{6}$ Department of Medicine and Department of Health Research Methods, Evidence \& Impact, McMaster University, Canada

${ }^{7}$ Department of Paediatrics and Paediatric Infectious Diseases, Institute of Child's Health, Sechenov First Moscow State Medical University (Sechenov University), Moscow, Russia

${ }^{8}$ Inflammation, Repair and Development Section, National Heart and Lung Institute, Faculty of Medicine, Imperial College London, London, United Kingdom

${ }^{9}$ Peter Gorer Department of Immunobiology, School of Immunology and Microbial Sciences, King's College London, London, UK

${ }^{10}$ Children's Allergy Service, Evelina London, Guy's and St Thomas' Hospital, London, UK

${ }^{11}$ Department of Allergy and Clinical Immunology, Royal Brompton and Harefield NHS Foundation Trust, London, UK

${ }^{12}$ Asthma UK Centre in Allergic Mechanisms of Asthma, London, UK

\section{Corresponding Author:}

Jon Genuneit

Pediatric Epidemiology

Department of Pediatrics

Medical Faculty, Leipzig University

Liebigstr. 20a, Haus 6

D-04103 Leipzig

GERMANY

Jon.genuneit@medizin.uni-leipzig.de

\section{*Full list of contributors:}

Cezmi A. Akdis ${ }^{13}$, Alberto Alvarez-Perea ${ }^{14}$, Montserrat Alvaro Lozano ${ }^{15}$, Riccardo Asero ${ }^{16}$, Barbara Ballmer-Weber ${ }^{17,18}$, Domingo Barber ${ }^{19,20}$, Simona Barni2 ${ }^{21}$, Giovanni Battista Pajno ${ }^{22}$, Kirsten Beyer $^{23}$, Carsten Bindslev-Jensen ${ }^{24}$, Helen Brough $^{25,26,27}$, Betul Buyuktiryaki ${ }^{28}$, Lucila Camargo Lopes de Oliveira ${ }^{29}$, Antonella Cianferoni ${ }^{30}$, R Sharon Chinthrajah ${ }^{31,32}$, Stefano Del Giacco ${ }^{33}$, Audrey DunnGalvin ${ }^{34,35,36}$, Bernadette Eberlein ${ }^{37}$, Motohiro Ebisawa ${ }^{38}$, Philippe Eigenmann ${ }^{39}$, Thomas Eiwegger $^{40,41,42}$, Margaretha Faber ${ }^{43}$, Montserrat Fernandez-Rivas ${ }^{44}$, Mary Feeney ${ }^{26,27}$, Helen Fisher ${ }^{27}$, Mattia Giovannini ${ }^{45}$, Susanne Halken ${ }^{46}$, Karin Hoffmann-Sommergruber ${ }^{47}$, Hannah Jaumdally ${ }^{27}$, Christina $\mathrm{J}_{\text {Jones }}{ }^{48}$, Rebecca Knibb ${ }^{49}$, Edward $\mathrm{Knol}^{50}$, George N Konstantinou ${ }^{51}$, Marta Krawiec ${ }^{26,27}$, Susanne Lau $^{52}$, Lina Mayorga ${ }^{53}$, M. Andreina Marques-Mejias ${ }^{26,27}$, Rosan Meyer ${ }^{54}$, Beatriz Moya ${ }^{55}$, Charlotte G Mortz $^{24}$, Antonella Muraro ${ }^{56}$, Kari Nadeau ${ }^{31,32}$, Caroline Nilsson ${ }^{57,58}$, Liam O’Mahony ${ }^{59}$, Nikolaos G. Papadopoulos $^{60,61}$, Kirsten Perrett ${ }^{62}$, Alexandre Piletta-Zanin ${ }^{39}$, Marcia Podestà ${ }^{63}$, Lars K. Poulsen ${ }^{64}$, Cristian Ricci ${ }^{1}$, Graham Roberts ${ }^{65,66}$, Hugh Sampson ${ }^{67}$, Sylwia Smolińska ${ }^{68}$, Jürgen Schwarze ${ }^{69}$, Eva Untersmayr $^{70}$, Ronald Van Ree ${ }^{71}$, Carina Venter ${ }^{72}$, Brian P. Vickery ${ }^{73}$, Berber Vlieg-Boerstra ${ }^{74}$

${ }^{13}$ Swiss Institute of Allergy and Asthma Research 
14 Hospital General Universitario Gregorio Marañón, Madrid, Spain

15 Pediatric Allergy and Clinical Immunology Service, Hospital Sant Joan de Déu, Barcelona

${ }^{16}$ Clinica San Carlo, Paderno Dugnano, Italy

17 Allergy Unit, Department of Dermatology, University Hospital of Zurich, Zurich, Switzerland; Faculty of Medicine, University of Zurich, Zurich, Switzerland

18 Clinic for Dermatology and Allergology, Kantonsspital St Gallen, St Gallen, Switzerland.

19 School of Medicine, Institute for Applied Molecular Medicine (IMMA), Universidad CEU San Pablo, Madrid, Spain.

20 Instituto de Salud Carlos III, Madrid, Spain

21 Meyer Children's University Hospital, Florence, Italy

22 Pediatric Unit, Policlinico Hospital. University of Messina-Italy

23 Charité Universitätsmedizin Berlin, Germany

24 Odense University Hospital, Odense Denmark

25 Evelina London Childrens Hospital, London, UK

26 Guy's and St Thomas' Hospital London, UK

27 King's College London, UK

${ }^{28}$ Koc University Hospital, Istanbul, Turkey

29 Federal University of São Paulo, Brazil

30 Department of Pediatrics, Division of Allergy and Immunology, The Children's Hospital of Philadelphia, PA, USA

31 Sean N Parker Center for Allergy and Asthma Research, Stanford University, USA

32 Pulmonary, Allergy, Critical Care Medicine, Department of Medicine, Stanford University, USA

33 Department of Medical Sciences and Public Health, University of Cagliari, Italy

34 University College Cork, Ireland

35 Anaphylaxis Ireland (CEO)

36 Sechenov University,Moscow

37 Department of Dermatology and Allergy BiedersteinTechnische Universität München

38 Department of Allergy, Clinical Research Center for Allergy and Rheumatology, Sagamihara National Hospital, Kanagawa, Japan

39 University Hospitals of Geneva, Switzerland

40 The Hospital for Sick Children, Toronto, Ontario

${ }^{41}$ University of Toronto, Ontario

${ }^{42}$ University Hospital of St. Pölten, Karl Landsteiner University of Health Sciences

43 University of Antwerp, Department of Immunology and Allergology, Antwerp, Belgium

44 Allergy Dept., Hospital Clinico San Carlos, Universidad Complutense, IdISSC, Madrid, Spain

45 Meyer Children's University Hospital, Florence, Italy 
46 Hans Christian Andersen Children's Hospital, Odense University Hospital

47 Medical University of Vienna, Austria

48 University of Surrey, Guildford, UK

49 Department of Psychology, Aston University, Birmingham, UK.

50 Departments of Immunology and Dermatology/Allergology, University Medical Center Utrecht, Utrecht, the Netherlands

${ }^{51}$ Department of Allergy and Clinical Immunology General Military Training Hospital, Thessaloniki, Greece

52 Universitaetsmedizin Berlin, Pediatric Respiratory Medicine, Immunology and Intensive Care Medicine, Germany

53 Biomedical Research Institute and University Hospital of Málaga

54 Imperial College London UK

55 Department of Allergy, Hospital Universitario 12 de Octubre, Madrid, Spain

${ }^{56}$ Food Allergy Referral Centre, Department of Woman and Child Health, Padua University hospital , Padua , Italy

57 Department of Clinical Science and Education, Södersjukhuset, Karolinska Institutet, Stockholm, Sweden

58 Sachs' Children and Youth Hospital, Södersjukhuset, Stockholm, Sweden

59 University College Cork, Cork, Ireland

60 Allergy Department, 2nd Pediatric Clinic, University of Athens, Greece

${ }^{61}$ Division of Infection, Immunity \& Respiratory Medicine, University of Manchester, UK

62 Murdoch Children's Research Institute, Melbourne, Australia

63 European Federation of Allergy and Airways Diseases Patient's Associations, Belgium

64 Copenhagen University Hospital, Denmark

${ }^{65}$ David Hide Centre, St Mary's Hospital, Isle of Wight, United Kingdom

66 University of Southampton, Southampton, United Kingdom

67 Department of Pediatrics at the Icahn School of Medicine at Mount Sinai

68 Wroclaw Medical University, Department of Clinical Immunology, Wroclaw, Poland

69 The University of Edinburgh, Edinburgh, UK

70 Institute of Pathophysiology and Allergy Research, Center for Pathophysiology, Infectiology and Immunology, Medical University of Vienna

71 Amsterdam University Medical Centers, Location AMC, The Netherlands

72 Children's Hospital Colorado, University of Colorado, Colorado, USA

73 Emory University and Children's Healthcare of Atlanta

74 OLVG Hospital, Amsterdam, the Netherlands

Conflict of interest statement: a separate COI form has been submitted due to the long list of authors.

Abstract (237 words) 
Background: The European Academy of Allergy and Clinical Immunology (EAACI) is in the process of updating the guidelines on the diagnosis and management of food allergy. The existing guidelines are based on a systematic review of the literature until $30^{\text {th }}$ September 2012. Therefore, a new systematic review must be undertaken to inform the new guidelines. This systematic review aims to assess the accuracy of index tests to support the diagnosis of IgE-mediated food allergy.

Methods: The databases Cochrane CENTRAL (Trials), MEDLINE (OVID) and Embase (OVID) will be searched for diagnostic test accuracy studies from $1^{\text {st }}$ October 2012 to $30^{\text {th }}$ June 2021. Inclusion and exclusion criteria will be used to select appropriate studies. Data from these studies will be extracted and tabulated, and then reviewed for risk of bias and applicability using the QUADAS-2 tool. All evaluation will be done in duplicate. Studies with a high risk of bias and low applicability will be excluded. Meta-analysis will be performed if there are three or more studies of the same index test and food.

Results: A protocol for the systematic review and meta-analyses is presented and was registered using Prospero prior to commencing the literature search.

Discussion: Oral food challenges are the reference standard for diagnosis but involve considerable risks and resources. This protocol for systematic review aims to assess the accuracy of various tests to diagnose food allergy, which can be useful in both clinical and research settings.

Keywords: Food allergy, IgE-mediated, diagnosis, diagnostic tests, specific IgE, skin prick test, componentresolved diagnostics, basophil activation test, mast cell activation test

Key message: This is the protocol for the systematic review of the literature which will inform the update of the Food Allergy Guidelines of the European Academy of Allergy and Clinical Immunology (EAACI).

\section{Background}

The global prevalence of food allergy is about $10 \%$ with an increase in its incidence in the last 20-30 years ${ }^{1,2}$. Evidence suggests that allergies to food are more common in Westernized countries, affecting children more than adults ${ }^{3}$. Any food can be a potential allergen; however, a large proportion of food allergies worldwide can be accounted for by nuts, milk, eggs, shellfish and wheat ${ }^{4}$. Different foods act as the most common allergens in different countries; while peanut allergies are relatively common in the UK, USA, Canada and Australia, they are very rarely seen in Asia, excluding Japan. Overall, milk and eggs appear to be the most common allergens in young children in the UK and most parts of Europe, USA, Canada, Asia and Australia ${ }^{5}$.

Food allergies can be classified as immunoglobulin E ( $\operatorname{IgE}$ ) mediated and non-IgE mediated, with the former being the most common and the focus of this systematic review. IgE mediated food allergies usually induce immediate reactions, i.e. reactions that occur up to 2 hours (usually a few minutes) after exposure to the allergen, and these can be severe, and sometimes life threatening. By contrast, non-IgE mediated allergies prompt a delayed response, with symptoms taking up to two days to evolve, or manifest chronically ${ }^{6}$. Clinical manifestations of food allergy include skin, gastrointestinal (GI) and respiratory reactions, with skin reactions being the most prevalent and presenting as urticaria, angioedema and erythema ${ }^{4,7}$. GI symptoms include abdominal pain, diarrhoea, nausea and vomiting. Rhinorrhoea, nasal obstruction, bronchospasms and oedema of the larynx are possible respiratory symptoms ${ }^{2}$. Allergic reactions can vary in severity, ranging from local reactions such as tingling in the mouth to anaphylaxis, a severe life-threatening allergic reaction affecting breathing or circulation ${ }^{4,8}$.

The diagnosis of IgE-mediated food allergy is usually based on the clinical presentation and evidence of $\operatorname{IgE}$ sensitisation to the specific allergen, as documented by a positive skin prick test (SPT) or serum specific $\operatorname{IgE}^{9}$. The reference standard is the oral food challenge (OFC), in which the suspected allergen is administered orally in gradually increasing doses until either a reaction occurs or all doses are tolerated. OFC are resource intensive as they must be conducted in a medical setting due to the risk of anaphylaxis. The results can subsequently be used to confirm the diagnosis, to assess tolerability in people with a confirmed allergy, or to detect the reaction threshold. In cases with a recent history of an allergic reaction, detectable IgE specific to the culprit allergen can be enough to confirm the diagnosis, dispensing oral food challenge. 
Several tests have been suggested as alternatives for OFC. While SPT and $\operatorname{sgE}$ confirm presence of $\operatorname{IgE}$ antibodies to a particular food (sensitisation) they do not necessarily correlate to a clinical reaction, with approximately half of children sensitised able to tolerate the food without reaction. These routine tests therefore generally have high sensitivity but poor specificity to clinical food allergy. Increasing magnitude of these tests are associated with increased risk of clinical reaction, and thresholds with high probability of food allergy have been identified for some foods (e.g. for peanut: SPT $>=8 \mathrm{~mm}$ or $\operatorname{sIgE}>=15$ have $95 \% \mathrm{PPV}$ ) which negate the need for OFC in some settings. Reported thresholds vary in the literature, likely due to differences in study design and patient characteristics. Component-resolved diagnosis (CRD) refers to the determination of specific IgE levels to specific proteins in food ${ }^{10}$. Additional tests include the basophil activation test (BAT) and the mast cell activation test (MAT); however these are currently not used in routine clinical settings. CRD, BAT and MAT are emerging tests with early studies suggesting that they offer an improvement on sensitivity and specificity than traditional SPT or sIgE tests.

The European Academy of Allergy and Clinical Immunology is currently in the process of updating their food allergy guidelines. Thus, a systematic review of existing literature will be carried out to inform the new guidelines. The systematic review aims to assess the diagnostic accuracy measured by sensitivity and specificity of index tests for IgE-mediated food allergy compared to the standard OFC. Furthermore, comparison among index tests will be conducted if sufficient evidence is available.

\section{Methods}

Search strategy

The following databases will be searched:

- Cochrane CENTRAL (Trials)

- MEDLINE (OVID)

- Embase (OVID)

These databases will be searched for entries between the period of $1^{\text {st }}$ October 2012 to the $31^{\text {st }}$ May 2021. This aligns with the end date of the search entries used in a previous systematic review on the same subject ${ }^{11}$. Studies which were published earlier and included in the previous systematic review will be evaluated for inclusion in the current systematic review.

Forward and backward citation analysis will be used in all included studies to pinpoint other relevant studies. Any additional studies discovered by the review team who are experts in the field may be included. Only published, peer-reviewed full reports will be included.

\section{Condition/domain being studied}

The main domain being investigated is IgE-mediated food allergy. We will explore any food allergen. Food allergens will be ranked by the frequency of available studies and index test, with the most commonly investigated allergen prioritised, if necessary. This is because we will assume that more studies will raise more high-quality studies, thus firming evidence. Next, the more recently developed tests (CRD, BAT, MAT) will be prioritised according to the frequency of reports. The previous EAACI Food Allergy Guideline paper has already given recommendations for SPT and specific IgE, and we aim to summarise novel evidence on SPT and specific IgE if this seems warranted.

\section{Population}

Studies of children and adults, irrespective of age, with suspected IgE-mediated allergy to any food will be considered.

\section{Interventions}

Any index tests to diagnose food allergy in the included studies will be reported. Priority will be placed on the more recently developed test strategies of specific IgE to allergen components, BAT and MAT as described above. 


\section{Comparator}

The comparator to define the accuracy for immediate-type food allergy diagnosis will be OFC, including both open food challenge or double-blind placebo-controlled food challenge, performed in at least a proportion of study participants.

\section{Inclusion criteria}

Studies fulfilling the following criteria will be included:

Studies containing sufficient data to calculate sensitivity and specificity, to get a measure of the diagnostic accuracy.

Diagnostic test studies with any sequence of index test and reference standard test performed and with any algorithm or procedure of participant selection. These characteristics will be part of the risk of bias assessment and evaluation of reasons for heterogeneity as laid out below.

\section{Exclusion criteria}

Studies fulfilling the following criteria will be excluded:

- Conference abstracts

- Narrative reviews, editorials and correspondence

- Systematic reviews (reference lists will first be scanned for relevant original articles)

- Qualitative studies

- Case reports and case series

- Animal studies

- Studies in which allergies are defined based on sensitization tests alone without a history following ingestion.

- Studies may be excluded based on risk of bias and applicability

Each study will be screened by two reviewers independently based on the title and abstract according to the inclusion and exclusion criteria (described below). If there are any discrepancies between the reviewers, a third reviewer will adjudicate. When the studies have been selected, the full texts will be reviewed to confirm they meet the inclusion criteria. Data will be extracted from these studies and the quality will be assessed for risk of bias and applicability. Studies which satisfy the inclusion criteria but are not in English will be translated prior to data extraction and quality assessment.

Data extraction and management

The details that will be collected from the selected studies are represented in Box $\mathbf{2}$. Data extraction will be conducted by two independent reviewers and subsequently recorded using COVIDENCE. Any discrepancies will be resolved by a third reviewer.

Quality assessment (risk of bias)

All included studies will be assessed for risk of bias and their applicability based on the QUADAS 2 instrument ${ }^{12}$. The four key domains covering patient selection, index test, reference standard (comparator), and flow and timing will be evaluated. Any studies deemed to have a high risk of bias i.e. scoring 'high' for three or more of the four risk of bias domains, or concerns regarding applicability i.e. scoring 'high' for two or more of the three applicability domains will be excluded from further meta-analyses.

\section{Strategy for data synthesis}

When possible, data extracted for sensitivity and specificity will be analysed using random effects metaanalysis using hierarchical summary receiver operating characteristic (ROC) curve analysis in case of three or more studies for a given combination of index test and food. 
The GRADE approach will be used to assess heterogeneity ${ }^{13,14}$, and will not solely rely on statistical methods such as the $\mathrm{I}^{2}$ statistic. Depending on the included studies, the following reasons for heterogeneity will be discussed:

- Multiple definitions of target condition

- Multiple thresholds of test positivity for index and/or comparator

- Age of the participants

- Origin of study population as indication for patient spectrum/pre-test probability

- All domains of risk of bias of the included studies

The GRADE approach will be used to evaluate the certainty of the body of evidence.

Sensitivity analysis

Sensitivity analysis may be carried out if three or more studies are retained for a given combination of index test and food allergen following exclusion of studies which are suspected to contribute to heterogeneity or limiting to studies with low risk of bias, i.e. studies with 'low' in all four risk of bias domains of the QUADAS-2.

\section{Dissemination plans}

The final report will be published in one of the scientific journals affiliated with EAACI and will be used to inform the update of the EAACI Guidelines on Food Allergy. The findings of the systematic review will be presented at scientific conferences, namely the EAACI annual congress and at the EAACI Food Allergy and Anaphylaxis Meeting. A summary of the findings will be made available in lay language on the EAACI website.

\section{Discussion}

The EAACI Food Allergy Guidelines need to be updated, in particular the section on diagnostic tests for food allergy. The systematic review of the literature on diagnostic tests that informed the current EAACI Food Allergy Guidelines was conducted prior to 2014, thus a new systematic review is needed, especially since emerging tests such as CRD, MAT and BAT were not included in the previous review. We report herein the protocol for a systematic review of the literature, as registered at PROSPERO (CRD42021259186), and provide a description of the rationale and methods chosen for the review.

OFC is the reference-standard diagnostic test for food allergies; however, there are considerable risks involved. OFC involve ingesting the suspected allergen, which is associated with a risk of allergic reaction and may result in a life-threatening event. As a result, OFC must be conducted in a controlled environment with intensive care facilities in easy reach, and not all clinical settings can offer this service. Additionally, this risk of reaction may lead to significant anxiety in patients and their families, therefore a reliable and cost-effective alternative to OFC is needed. Since the previous guideline was published, newer tests such as CRD have entered mainstream use in Europe and other regions. Also, studies assessing the efficacy of novel diagnostic tests such as BAT and MAT have been published. All of these new tests must be reviewed to assess whether they are acceptable diagnostic tools for use in routine clinical practice. For more established allergy tests, such as SPT and SIgE, new studies have been performed since the previous review which may require updating of the previous recommendation. We aim to evaluate the diagnostic test accuracy of any index test, from SPT, sIgE to extracts, sIgE to individual allergens, sIgE to allergen peptides, BAT and MAT. Thus, we do not include search terms for the index test. We apply a specific filter for diagnostic test accuracy studies, though, which implicitly captures studies on diagnostics and/or tests. We only include search terms for "challenge" as part of the common terminology for the comparator, i.e. oral food challenge, to have a sensitive search regarding the comparator test.

The population of interest will include all ages and diverse clinical settings and geographical locations. We deliberately do not include search terms to specify the target population and studies that do not have evidence from all populations will be included in the review and judged for risk of bias. We expect to encounter very 
few if any diagnostic test accuracy studies conducted in animals; however, we will refrain from specifying search terms that will exclude animal studies because of the risk of falsely excluding studies on animal allergen sources. Certain food allergies are more prevalent in different areas. As such, we will include studies focussing on any food, to be inclusive to all populations, although we anticipate that there will be more studies on milk, egg and peanut allergies.

Although the gold standard is DBPCFC, we will also include studies where open oral food challenges are performed and allow for the inclusion of studies where a small proportion of patients were diagnosed without an oral food challenge due to previous severe or anaphylactic reactions. Studies focusing only on IgE sensitisation will be excluded, as some patients who experience sensitisation to foods are not necessarily allergic. It is important to note that the comparator reference standard test is oral food challenge and as such, the systematic review will inform on the diagnostic accuracy of potential alternative tests. However, the systematic review will not clarify which of these tests is best used in subjects in whom the oral food challenge is contraindicated.

As outcomes we are assessing sensitivity and specificity as measures of diagnostic test accuracy. The positive and negative predictive value of the test, which depends on the prevalence of the given food allergy in the population, is not within the scope of this systematic review. The details collected as part of the quality assessment and risk of bias will allow us to select high-quality studies at low risk of bias. The planned sensitivity analyses on these features may help to identify factors that influence the performance of the test and the modulation of the identified diagnostic cut-offs. If sufficient high quality studies are identified, the planned meta-analyses will provide evidence which may help to reduce the number of patients that have to undergo an OFC procedure.

Tables and Figures:

Box 1. Question central to the systematic review in the PICO format

Box 2. Data to be extracted from the full-length articles selected for the systematic review

Table 1. Search terms used in the systematic review. 
"food hypersensit:".mp OR "food allerg:".mp OR "food sensit:".mp OR "Food-dependent exercise-induced anaphylaxis".mp OR "cow?? adj2 allerg:".mp OR "milk adj2 allerg:".mp OR "peanut? adj2 allerg:".mp OR "egg? adj2 allerg:".mp OR "nut? adj2 allerg:".mp OR "hazelnut? adj2 allerg:".mp OR "walnut? adj2 allerg:".mp OR "pistachio? adj2 allerg:".mp OR "almond? adj2 allerg:".mp OR "brazil adj2 allerg:".mp OR "macadamia adj2 allerg:".mp OR "pecan adj2 allerg:".mp OR "cashew? adj2 allerg:".mp OR "sesame adj2 allerg:".mp OR "soy? adj2 allerg:".mp OR "lentil? adj2 allerg:".mp OR "pea? adj2 allerg:".mp OR "chickpea? adj2 allerg:".mp OR "rice adj2 allerg:".mp OR "celery adj2 allerg:".mp OR "peach adj2 allerg:".mp OR "apple? adj2 allerg:".mp OR "wheat adj2 allerg:".mp OR "gluten adj2 allerg:".mp OR "mustard adj2 allerg:".mp OR "meat adj3 allerg:".mp OR "beef adj2 allerg:".mp OR "pork adj2 allerg:".mp OR "alpha-gal adj2 allerg:".mp OR "fish adj2 allerg:".mp OR "seafood adj2 allerg:".mp OR "shrimp? adj2 allerg:".mp challeng:.mp. OR provocation:.mp. OR anaphylaxis.mp sensitiv:.mp. OR "predictive value:".mp. OR accurac:.tw.

1 AND 2 AND 3

MEDLINE: limit 3 to

$\mathrm{dt}=20121001-20210531$

EMBASE: limit 3 to

$\mathrm{dd}=20121001-20210531$
Clinical condition Most likely to be captured by the first four general search terms but augmented by terms specific for foods in combination with terms for allergy in varying order (e.g. "cashew allergy", "cashew nut allergy", "allergic to cashew" will all be captured by "cashew adj2 allerg:")

broad term for reference standard (i.e. oral food challenge or provocation) or anaphylaxis in instances when oral challenges were not conducted

McMaster HiRU filter with best balance of sensitivity and specificity to identify diagnostic test accuracy studies

Date since end of the search from previous systematic review 


\section{References:}

1. Gupta RS, Warren CM, Smith BM, et al. The Public Health Impact of Parent-Reported Childhood Food Allergies in the United States. Pediatrics 2018;142.

2. Baseggio Conrado A, Ierodiakonou D, Gowland MH, Boyle RJ, Turner PJ. Food anaphylaxis in the United Kingdom: analysis of national data, 1998-2018. BMJ 2021;372:n251.

3. Sicherer SH, Sampson HA. Food allergy: A review and update on epidemiology, pathogenesis, diagnosis, prevention, and management. J Allergy Clin Immunol 2018;141:41-58.

4. Lopez CM, Yarrarapu SNS, Mendez MD. Food Allergies. StatPearls. Treasure Island (FL)2021.

5. Tham EH, Leung DYM. How Different Parts of the World Provide New Insights Into Food Allergy. Allergy Asthma Immunol Res 2018;10:290-9.

6. Walsh J, Meyer R, Shah N, Quekett J, Fox AT. Differentiating milk allergy (IgE and non-IgE mediated) from lactose intolerance: understanding the underlying mechanisms and presentations. Br J Gen Pract 2016;66:e609-11.

7. Waserman S, Begin P, Watson W. IgE-mediated food allergy. Allergy Asthma Clin Immunol 2018;14:55.

8. Dribin TE, Schnadower D, Spergel JM, et al. Severity grading system for acute allergic reactions: A multidisciplinary Delphi study. J Allergy Clin Immunol 2021.

9. Foong RX, Dantzer JA, Wood RA, Santos AF. Improving Diagnostic Accuracy in Food Allergy. J Allergy Clin Immunol Pract 2021;9:71-80.

10. Callery EL, Keymer C, Barnes NA, Rowbottom AW. Component-resolved diagnostics in the clinical and laboratory investigation of allergy. Ann Clin Biochem 2020;57:26-35.

11. Soares-Weiser K, Takwoingi Y, Panesar SS, et al. The diagnosis of food allergy: a systematic review and meta-analysis. Allergy 2014;69:76-86.

12. Whiting PF, Rutjes AW, Westwood ME, et al. QUADAS-2: a revised tool for the quality assessment of diagnostic accuracy studies. Ann Intern Med 2011;155:529-36.

13. Guyatt GH, Oxman AD, Vist GE, et al. GRADE: an emerging consensus on rating quality of evidence and strength of recommendations. BMJ 2008;336:924-6.

14. Schunemann HJ, Oxman AD, Brozek J, et al. Grading quality of evidence and strength of recommendations for diagnostic tests and strategies. BMJ 2008;336:1106-10. 